\title{
The feasibility score for the Laparoscopic Appendicitis Score
}

\author{
Jenneke T. H. Hamminga $\cdot$ Jan Willem Haveman
}

Received: 19 March 2013/Accepted: 10 April 2013/Published online: 4 May 2013

(C) Springer Science+Business Media New York 2013

We appreciate the comment of Liu and Lu on our article. In response to their first concern about the fecaliths, we agree with the statement that fecaliths are associated with appendicitis and perforation of the appendix. We are unsure whether the presence of fecaliths should be an item in the Laparoscopic Appendicitis Score (LAPP score). Therefore, we retrospectively analyzed all high-definition videos we took from the 25 normal appendices.

As described in our Methods section, all appendices were thoroughly evaluated by the surgeon, and this was captured on video. In none of the 25 normal appendices a fecalith was present. Therefore, in our opinion, a fecalith in a normal appendix is very rarely seen and probably not relevant. However, we believe that in our planned large multicenter study, we should prospectively register the presence of fecaliths.

We agree with the authors that a thickened peritoneum around the appendix caused by other intraabdominal pathology should not be a reason for an appendectomy. In our view, it is not difficult for surgeons to differentiate a thickened appendix or mesoappendix from appendicitis or from peritonitis caused by other pathology.

We do not agree with the last concern raised by Liu and Lu. In our view, there is no literature on the interval between the onset of symptoms and the intraoperative appearance of the appendix. We speculate that in case the patient has right iliac fossa pain from appendicitis, there is peritonitis and thus the peritoneum or appendix is thickened. We saw 17 of our patients within $12 \mathrm{~h}$ of their initial complaints, and we did not notice any difference between the appearance of their appendices and those of patients with a longer duration of symptoms. Notably, 5 of these 17 appendices were necrotic $(29 \%)$, and 7 of the patients had pus around their appendix (41\%). In comparison, for all the patients with appendicitis $(n=109)$, this was a $28 \%$ rate for necrosis and a $53 \%$ rate for pus.

Disclosures Jenneke T. H. Hamminga and Jan Willem Haveman have no conflicts of interest or financial ties to disclose.

For the Apple Study Group; Appendicitis and Laparoscopic evaluation.

J. T. H. Hamminga · J. W. Haveman $(\square)$

Department of Surgery, University Medical Center Groningen,

University of Groningen, Hanzeplein 1, 9700 RB Groningen,

The Netherlands

e-mail: janwillemhaveman@gmail.com 